\title{
Bernoulli Identities and Combinatoric Convolution Sums with Odd Divisor Functions
}

\author{
Daeyeoul Kim ${ }^{1}$ and Yoon Kyung Park ${ }^{2}$ \\ ${ }^{1}$ National Institute for Mathematical Sciences, Yuseong-Daero 1689-Gil, Yuseong-Gu, Daejeon 305-811, Republic of Korea \\ ${ }^{2}$ School of Mathematics, Korea Institute for Advanced Study, 85 Hoegiro, Dongdaemungu, Seoul 130-722, Republic of Korea \\ Correspondence should be addressed to Yoon Kyung Park; ykpark@math.kaist.ac.kr
}

Received 18 October 2013; Accepted 2 December 2013; Published 12 January 2014

Academic Editor: Junesang Choi

Copyright (c) 2014 D. Kim and Y. K. Park. This is an open access article distributed under the Creative Commons Attribution License, which permits unrestricted use, distribution, and reproduction in any medium, provided the original work is properly cited.

We study the combinatoric convolution sums involving odd divisor functions, their relations to Bernoulli numbers, and some interesting applications.

\section{Introduction}

The Bernoulli polynomials $B_{k}(x)$, which are usually defined by the exponential generating function

$$
\frac{t e^{x t}}{e^{t}-1}=\sum_{k=0}^{\infty} B_{k}(x) \frac{t^{k}}{k !}
$$

play an important role in different areas of mathematics, including number theory and the theory of finite differences. It is well known that $B_{k}=B_{k}(0)$ are rational numbers. It can be shown that $B_{2 k+1}=0$ for $k \geq 1$ and is alternatively positive and negative for even $k$. The $B_{k}$ are called Bernoulli numbers. Let $\mathbb{N}$ denote the set of positive integers. Further, let $N, d, k \in \mathbb{N}$, where $s, r \in \mathbb{N} \cup\{0\}$. Throughout this paper, we define divisor functions as follows:

$$
\begin{gathered}
\sigma_{s}(n)=\sum_{d \mid n} d^{s}, \quad \sigma_{s, r}(n ; m)=\sum_{\substack{d \mid n \\
d \equiv r \bmod m}} d^{s}, \\
\widetilde{\sigma}_{s}(n)=\sum_{d \mid n}(-1)^{d-1} d^{s}, \quad \sigma_{s}^{*}(n):=\sum_{\substack{d \mid n \\
n / d \text { odd }}} d^{s} .
\end{gathered}
$$

We also make use of the following convention:

$$
\sigma_{s}(n)=0 \quad \text { if } n \notin \mathbb{Z} \text { or } n \leq 0 .
$$

Ramanujan [1] proved that

$$
\begin{aligned}
& \sum_{m=1}^{n-1} \sigma_{1}(m) \sigma_{1}(n-m)=\frac{1}{12}\left(5 \sigma_{3}(n)+(1-6 n) \sigma_{1}(n)\right), \\
& \sum_{m=1}^{n-1} \sigma_{1}(m) \sigma_{3}(n-m) \\
& \quad=\frac{1}{240}\left[21 \sigma_{5}(n)+(10-30 n) \sigma_{3}(n)-\sigma_{1}(n)\right]
\end{aligned}
$$

using elementary arguments.

Let $\mathfrak{H}$ be the complex upper half plane $\{z \in \mathbb{C}: \operatorname{Im}(z)>$ $0\}$ and let $q$ be $e^{2 \pi i z}$ for $z \in \mathfrak{H}$. Denote $\eta(z)$ by the Dedekind $\eta$-function

$$
\eta(z)=q^{1 / 24} \prod_{n \geq 1}\left(1-q^{n}\right)
$$

and $c_{6}(n)$ by the $n$th coefficient of $(\eta(z) \eta(2 z) \eta(3 z) \eta(6 z))^{2}$. Alaca and Williams [2] proved that

$$
\begin{aligned}
& \sum_{\substack{(l, m) \in \mathbb{N}^{2} \\
2 l+3 m=n}} \sigma_{1}(l) \sigma_{1}(m) \\
& =\frac{1}{120} \sigma_{3}(n)+\frac{1}{30} \sigma_{3}\left(\frac{n}{2}\right)
\end{aligned}
$$




$$
\begin{aligned}
& +\frac{3}{40} \sigma_{3}\left(\frac{n}{3}\right)+\frac{3}{10} \sigma_{3}\left(\frac{n}{6}\right)+\left(\frac{1}{24}-\frac{n}{12}\right) \sigma_{1}\left(\frac{n}{2}\right) \\
& +\left(\frac{1}{24}-\frac{n}{8}\right) \sigma_{1}\left(\frac{n}{3}\right)-\frac{1}{120} c_{6}(n) .
\end{aligned}
$$

It turns out that we need not only divisor functions but also the coefficients of certain modular functions. For other divisor functions, Hahn [3] showed that

$$
16 \sum_{m=1}^{n-1} \widetilde{\sigma}_{1}(m) \widetilde{\sigma}_{3}(n-m)=-\widetilde{\sigma}_{5}(n)+2(n-1) \widetilde{\sigma}_{3}(n)+\widetilde{\sigma}_{1}(n)
$$

and Glaisher [4-6] extended Besgue's formula by replacing $\sigma_{1}(n)$ in the convolution sum in (4) by other sums $\sigma^{*}(n)$; for example,

$$
\begin{aligned}
& 24 \sum_{m=1}^{n-1} \sigma_{1}^{*}(m) \sigma_{1}^{*}(n-m)=6 \sigma_{3}(n)-6 \sigma_{3}\left(\frac{n}{2}\right) \\
& \quad-6 n \sigma_{1}(n)+6 n \sigma_{1}\left(\frac{n}{2}\right) .
\end{aligned}
$$

Recently, the combinatorial convolution sum is studied [710]. In [10] Williams proved the following.

Proposition 1. Let $k, n \in \mathbb{N}$ and $n \geq 2$. Then

$$
\begin{array}{r}
\sum_{s=0}^{k-1}\left(\begin{array}{c}
2 k \\
2 s+1
\end{array}\right) \sum_{m=1}^{n-1} \sigma_{2 k-2 s-1}(m) \sigma_{2 s+1}(n-m) \\
=\frac{2 k+3}{4 k+2} \sigma_{2 k+1}(n)+\left(\frac{k}{6}-n\right) \sigma_{2 k-1}(n) \\
+\frac{1}{2 k+1} \sum_{j=2}^{k}\left(\begin{array}{c}
2 k+1 \\
2 j
\end{array}\right) B_{2 j} \sigma_{2 k+1-2 j}(n) .
\end{array}
$$

Cho et al. found out the linear sum for combinatorial convolution sum of $\sigma_{l}(m ; i, N)$ in [7].

Proposition 2. For $k, N \in \mathbb{N}$ and $N \geq 3$, one has

$$
\begin{aligned}
& \sum_{r=0}^{2 k}\left(\begin{array}{c}
2 k \\
r
\end{array}\right) \sum_{i=1}^{[(N-1) / 2]} \sum_{m=1}^{n-1} \sigma_{2 k-r}(m ; i, N) \sigma_{r}(n-m ; i, N) \\
& =\sigma_{2 k+1}^{*}(n ; N)-\frac{2}{N} n \sigma_{2 k-1}^{*}(n ; N) \\
& \quad-\frac{1}{N} \sum_{i=1}^{[(N-1) / 2]}(N-2 i) \sigma_{2 k}(n ; i, N) \\
& \quad-\frac{1+(-1)^{N}}{2}\left(\sigma_{2 k+1}^{*}\left(\frac{2 n}{N} ; 2\right)-\frac{2}{N} n \sigma_{2 k-1}^{*}\left(\frac{2 n}{N} ; 2\right)\right),
\end{aligned}
$$

where

$$
\begin{gathered}
\sigma_{r}(n ; i, N)=\sum_{\substack{d \mid n \\
n / d \equiv i(N)}} d^{r}-(-1)^{r} \sum_{\substack{d \mid n \\
n / d \equiv-i(N)}} d^{r}, \\
\sigma_{r}^{*}(n ; N)=\sum_{\substack{d \mid n \\
n / d \neq 0(N)}} d^{r}=\sigma_{r}(n)-\sigma_{r}\left(\frac{n}{N}\right) .
\end{gathered}
$$

Denote $\widetilde{B}_{k}$ by $\sum_{j=0}^{k}\left(\begin{array}{c}k \\ j\end{array}\right) 2^{j} B_{j}=2^{k} B_{k}(1 / 2)$. The generating function $2 t e^{t} /\left(e^{2 t}-1\right)$ of $\widetilde{B}_{k}$ is an even function and $\widetilde{B}_{k}$ is zero for all odd positive integer $k$. The aim of this paper is to study two combinatorial convolution sums of the analogous type of Proposition 2. When we write the convolution sums as linear sum of divisor function, in the result by Williams the coefficients are $B_{k}=B_{k}(0)$ and ours are $B_{k}(1 / 2)$. More precisely, we prove the following theorems.

Theorem 3. For $k, n \in \mathbb{N}$ and $n \geq 2$,

$$
\begin{aligned}
& \sum_{s=0}^{k-1}\left(\begin{array}{c}
2 k \\
2 s+1
\end{array}\right) \sum_{m=1}^{n-1} \sigma_{2 k-2 s-1,1}(m ; 2) \sigma_{2 s+1,1}(n-m ; 2) \\
& =2^{2 k-1} \sigma_{2 k+1}\left(\frac{n}{2}\right)+\frac{1}{4 k+2} \sum_{i=0}^{k}\left(\begin{array}{c}
2 k+1 \\
2 i+1
\end{array}\right) \\
& \times \widetilde{B}_{2 k-2 i} \sigma_{2 i+1,1}(n ; 2) .
\end{aligned}
$$

Equation (7) is a special case when $k=2$ for the following theorem because $\widetilde{B}_{4}=14 / 30$ and $B_{4}=-1 / 30$.

Theorem 4. For $k, n \in \mathbb{N}$ and $n \geq 2$,

$$
\begin{aligned}
\sum_{s=0}^{k-1}\left(\begin{array}{c}
2 k \\
2 s+1
\end{array}\right) \sum_{m=1}^{n-1} \widetilde{\sigma}_{2 k-2 s-1}(m) \widetilde{\sigma}_{2 s+1}(n-m) \\
=-\frac{1}{2} \widetilde{\sigma}_{2 k+1}(n)+\left(n-\frac{k}{2}\right) \widetilde{\sigma}_{2 k-1}(n) \\
\quad+\frac{1}{4 k+2} \sum_{i=0}^{k-2}\left(\begin{array}{c}
2 k+1 \\
2 i+1
\end{array}\right) \\
\quad \times\left(\widetilde{B}_{2 k-2 i}+\left(2^{2 k-2 i}-2\right) B_{2 k-2 i}\right) \sigma_{2 i+1}(n) \\
+\frac{1}{4 k+2} \sum_{i=0}^{k-2}\left(\begin{array}{c}
2 k+1 \\
2 i+1
\end{array}\right) \\
\quad \times\left(\widetilde{B}_{2 k-2 i}-2^{2 k-2 i} B_{2 k-2 i}\right) \widetilde{\sigma}_{2 i+1}(n) .
\end{aligned}
$$

Remark 5. The product of two modular forms is another modular form of bigger weight. The dimension $[(k+1) / 2]+1$ of space $M_{2 k+2}\left(\Gamma_{0}(2)\right)$ of modular forms on $\Gamma_{0}(2)$ is approximately linear for $k$ and the space $E_{2 k+2}\left(\Gamma_{0}(2)\right)$ generated by generating functions of divisor functions is clearly 2 as $k$ grows. More precisely speaking, for the Eisenstein series $G_{2 k+2}$ and $G_{2 k+2}^{*}$ which will be defined in Section 2

$$
\begin{aligned}
& G_{2 s+2}^{*}(z) G_{2 k-2 s}^{*}(z) \in M_{2 k+2}\left(\Gamma_{0}(2)\right) \\
& \quad=E_{2 k+2}\left(\Gamma_{0}(2)\right) \bigoplus S_{2 k+2}\left(\Gamma_{0}(2)\right),
\end{aligned}
$$


where $S_{2 k+2}\left(\Gamma_{0}(2)\right)$ is the space of cusp form of weight $2 k+2$ on $\Gamma_{0}(2)$ and it is orthogonal complement of $E_{2 k+2}\left(\Gamma_{0}(2)\right)$ in $M_{2 k+2}\left(\Gamma_{0}(2)\right)$. Since $E_{2 k+2}\left(\Gamma_{0}(2)\right)=\left\langle G_{2 k+2}, G_{2 k+2}^{*}\right\rangle$ and $S_{2 k+2}\left(\Gamma_{0}(2)\right)=\left\langle h_{1}, \ldots, h_{[(k+1) / 2]-1}\right\rangle$,

$$
\begin{aligned}
G_{2 s+2}^{*} & (z) G_{2 k-2 s}^{*}(z) \\
& =\alpha_{1} G_{2 k+2}(z)+\alpha_{2} G_{2 k+2}^{*}(z)+\sum_{r=1}^{[(k+1) / 2]-1} \beta_{r} h_{r}(z)
\end{aligned}
$$

for suitable constants $\alpha_{1}, \alpha_{2}, \beta_{1}, \ldots, \beta_{[(k+1) / 2]-1}$. On the other hand, Theorems 3 and 4 show that the combinatorial convolution sums are written as only divisor functions; that is,

$$
\begin{gathered}
\sum_{s=0}^{k-1}\left(\begin{array}{c}
2 k \\
2 s+1
\end{array}\right) G_{2 s+2}^{*}(z) G_{2 k-2 s}^{*}(z) \\
=\alpha_{1} G_{2 k+2}(z)+\alpha_{2} G_{2 k+2}^{*}(z) .
\end{gathered}
$$

The disappearance of $\beta_{1}, \ldots, \beta_{[(k+1) / 2]-1}$ is observed in Examples 17 and 18.

All calculations in Lemmas 6 and 7 and Theorems 9 and 10 are obtained by usins SAGE.

\section{Modular Forms}

In this section, we observe the convolution sums as a view point of generating functions of divisor functions.

The normalized Eisenstein series $G_{k}$ is defined by

$$
G_{k}(z)=-\frac{B_{k}}{2 k}+\sum_{n=1}^{\infty} \sigma_{k-1}(n) q^{n} .
$$

For the generating function of $\sigma_{k-1,1}(n)$ we denote

$$
\begin{aligned}
G_{k}^{*}(z) & :=G_{k}(z)-2^{k-1} G_{k}(2 z) \\
& =\frac{2^{k-1}-1}{2 k} B_{k}+\sum_{n=1}^{\infty} \sigma_{k-1,1}(n ; 2) q^{n} .
\end{aligned}
$$

Let $\Gamma$ be a finite index subgroup of $\operatorname{SL}(2, \mathbb{Z})$. The modular form $f(z)$ of weight $k$ on $\Gamma$ is a holomorphic function on $\mathfrak{H}$ such that

$$
f\left(\frac{a z+b}{c z+d}\right)=(c z+d)^{k} f(z) \quad \forall\left(\begin{array}{ll}
a & b \\
c & d
\end{array}\right) \in \Gamma
$$

for a positive integer $k$. The vector space over $\mathbb{C}$ of holomorphic modular forms of weight $k$ on $\Gamma$ is finite dimensional and is denoted by $M_{k}(\Gamma)$.

Note that $G_{k} \in M_{k}(\operatorname{SL}(2, \mathbb{Z}))$ (if $2 \mid k>2$ ) and $G_{k}^{*} \in$ $M_{k}\left(\Gamma_{0}(2)\right)$ (if $\left.2 \mid k \geq 2\right)$ for $\Gamma_{0}(2)=\left\{\left(\begin{array}{ll}a & b \\ c & d\end{array}\right) \in \operatorname{SL}(2, \mathbb{Z}): 2 \mid\right.$ $c$. Moreover, the product of two modular forms $f_{1}$ and $f_{2}$ of weights $k_{1}$ and $k_{2}$ is also modular form of weight $k_{1}+k_{2}$.

The $\Delta$ is the discriminant function

$$
\Delta(z)=\eta^{24}(z)=\sum_{n \geq 1} \tau(n) q^{n}
$$

with Ramanujan $\tau$-function as its coefficient. It is modular of weight 12 on $S L(2, \mathbb{Z})$.
Define the following two weight 14 modular forms $\Delta_{14,1}$ and $\Delta_{14,2}$ by using the Dedekind $\eta$-function defined in (5):

$$
\begin{gathered}
\Delta_{14,1}(z)=14\left(G_{6}^{*}(z)-5 G_{6}(z)\right) \eta^{8}(z) \eta^{8}(2 z) \\
=\sum_{N=1}^{\infty} c_{14,1}(N) q^{N} \\
\Delta_{14,2}(z)=24^{3}\left(G_{2}^{*}(z)\right)^{3} \eta^{8}(z) \eta^{8}(2 z)=\sum_{N=1}^{\infty} c_{14,2}(N) q^{N} .
\end{gathered}
$$

We get the lemma.

Lemma 6. Consider the following:

(1)

$$
\begin{aligned}
G_{2}^{*}(z) G_{10}^{*}(z)= & \frac{31}{5528} G_{12}(z)-\frac{227}{60808} G_{12}^{*}(z) \\
& +\frac{1365}{691} \Delta(z)+\frac{26880}{691} \Delta(2 z),
\end{aligned}
$$

(2)

$$
\begin{aligned}
G_{4}^{*}(z) G_{8}^{*}(z)= & \frac{17}{44224} G_{12}(z)-\frac{79}{221120} G_{12}^{*}(z) \\
& -\frac{203}{691} \Delta(z)-\frac{4928}{691} \Delta(2 z),
\end{aligned}
$$

(3)

$$
\begin{aligned}
\left(G_{6}^{*}(z)\right)^{2}= & \frac{1}{5528} G_{12}(z)-\frac{61}{348264} G_{12}^{*}(z) \\
& +\frac{85}{691} \Delta(z)+\frac{2560}{691} \Delta(2 z) .
\end{aligned}
$$

Proof. The functions $G_{2}^{*} G_{10}^{*}, G_{4}^{*} G_{8}^{*}$, and $\left(G_{6}^{*}\right)^{2}$ are modular functions of weight 12 on $\Gamma_{0}(2)$. Note that $M_{12}\left(\Gamma_{0}(2)\right)$ is a 4-dimensional vector space over $\mathbb{C}$ generated by $G_{12}(z), G_{12}^{*}(z), \Delta(z)$, and $\Delta(2 z)$ because $\operatorname{dim}_{\mathbb{C}}\left(M_{k}\left(\Gamma_{0}(2)\right)\right)=$ $[k / 4]+1$. Their $q$-expansions are as follows:

$$
\begin{aligned}
G_{12}(z)= & \frac{691}{65520}+q+2049 q^{2}+177148 q^{3} \\
& +4196353 q^{4}+48828126 q^{5}+O\left(q^{6}\right), \\
G_{12}^{*}(z)= & -\frac{1414477}{65520}+q+q^{2}+177148 q^{3}+q^{4} \\
& +48828126 q^{5}+O\left(q^{6}\right), \\
\Delta(z)= & q-24 q^{2}+252 q^{3}-1472 q^{4} \\
& +4830 q^{5}+O\left(q^{6}\right), \\
G_{2}^{*}(z) G_{10}^{*}(z)= & \frac{511}{6336}+\frac{87}{44} q+\frac{131}{44} q^{2}+\frac{9129}{11} q^{3} \\
& +\frac{866403}{44} q^{4}+\frac{2223801}{22} q^{5}+O\left(q^{6}\right),
\end{aligned}
$$




$$
\begin{aligned}
G_{4}^{*}(z) G_{8}^{*}(z)= & \frac{889}{115200}-\frac{47}{160} q+\frac{113}{160} q^{2}-\frac{2769}{40} q^{3} \\
& +\frac{354673}{160} q^{4}-\frac{7521}{80} q^{5}+O\left(q^{6}\right), \\
\left(G_{6}^{*}(z)\right)^{2}= & \frac{961}{254016}+\frac{31}{252} q+\frac{283}{252} q^{2}+\frac{2017}{63} q^{3} \\
& +\frac{123259}{252} q^{4}+\frac{36731}{42} q^{5}+O\left(q^{6}\right) .
\end{aligned}
$$

By comparing the above expansions with ones of $G_{2 s}^{*}(z)$ $G_{12-2 s}^{*}(z)$ for $s=1,2,3$, we get our result.

Lemma 7. Consider the following:

(1)

$$
\begin{aligned}
G_{2}^{*}(z) G_{12}^{*}(z)= & \frac{691}{174752} G_{14}(z)-\frac{1885739}{715609440} G_{14}^{*}(z) \\
& -\frac{891}{86} \Delta_{14,1}(z)-\frac{8525}{762} \Delta_{14,2}(z),
\end{aligned}
$$

(2)

$$
\begin{aligned}
G_{4}^{*}(z) G_{10}^{*}(z)= & \frac{31}{174752} G_{14}(z)-\frac{4769}{28834080} G_{14}^{*}(z) \\
& +\frac{81}{86} \Delta_{14,1}(z)+\frac{245}{254} \Delta_{14,2}(z),
\end{aligned}
$$

(3)

$$
\begin{aligned}
G_{6}^{*}(z) G_{8}^{*}(z)= & \frac{17}{349504} G_{14}(z)-\frac{5249}{110093760} G_{14}^{*}(z) \\
& -\frac{9}{86} \Delta_{14,1}(z)-\frac{25}{254} \Delta_{14,2}(z) .
\end{aligned}
$$

Proof. One can prove these by using a similar way to Lemma 6 and 4-dimensional vector space $M_{14}\left(\Gamma_{0}(2)\right)$ generated by $G_{14}, G_{14}^{*}, \Delta_{14,1}$, and $\Delta_{14,2}$ over $\mathbb{C}$ because their $q^{-}$ expansions are as follows:

$$
\begin{aligned}
G_{14}(z)= & -\frac{1}{24}+q+8193 q^{2}+1594324 q^{3} \\
& +67117057 q^{4}+1220703126 q^{5}+O\left(q^{6}\right) \\
G_{14}^{*}(z)= & \frac{8191}{24}+q+q^{2}+1594324 q^{3} \\
& +q^{4}+1220703126 q^{5}+O\left(q^{6}\right) \\
\Delta_{14,1}(z)= & q-64 q^{2}-1836 q^{3} \\
& +4096 q^{4}+3990 q^{5}+O\left(q^{6}\right) \\
\Delta_{14,2}(z)= & +64 q^{2}+1236 q^{3} \\
& +4096 q^{4}-57450 q^{5}+O\left(q^{6}\right) .
\end{aligned}
$$

Remark 8. (1) $\Delta(z)$ is the normalized Hecke eigenform on the full modular group of weight 12 :

$$
\begin{gathered}
\tau\left(p^{r+1}\right)=\tau\left(p^{r}\right) \tau(p)-p^{11} \tau\left(p^{r-1}\right) \quad \text { for } p \text { prime, } r \geq 1 \\
\tau(m n)=\tau(m) \tau(n) \quad \text { if } \operatorname{gcd}(m, n)=1
\end{gathered}
$$

(2) $\Delta_{14,1}(z)$ and $\Delta_{14,2}(z)$ are normalized newforms on $\Gamma_{0}(2)$ of weight 14 . The coefficients $c_{k, j}(n)(j=1,2)$ satisfy that

$$
\begin{gathered}
c_{14, j}\left(p^{r+1}\right)=c_{14, j}\left(p^{r}\right) c_{14, j}(p) \\
-p^{13} c_{14, j}\left(p^{r-1}\right) \quad \text { for } p \text { odd prime } p, r \geq 1, \\
c_{14, j}\left(2^{r}\right)=c_{14, j}(2)^{r} \quad \text { for } r \geq 0, \\
c_{14, j}(m n)=c_{14, j}(m) c_{14, j}(n) \quad \text { if } \operatorname{gcd}(m, n)=1 .
\end{gathered}
$$

Moreover, for the Atkin-Lehner involution $W_{2}=\left(\begin{array}{cc}0 & -1 \\ 2 & 1\end{array}\right)$,

$$
\left.\Delta_{14,1}\right|_{14} W_{2}=\Delta_{14,1},\left.\quad \Delta_{14,2}\right|_{14} W_{2}=-\Delta_{14,2}
$$

if we define the action $\gamma=\left(\begin{array}{ll}a & b \\ c & d\end{array}\right) \in \mathrm{GL}(2, \mathbb{Q})$ on the complex valued function $f$ as

$$
\left(\left.f\right|_{k} \gamma\right)(z)=\operatorname{det}(\gamma)^{k / 2}(c z+d)^{-k} f\left(\frac{a z+b}{c z+d}\right) .
$$

By the help of Lemmas 6 and 7 we get the formulae for each convolution sum.

Theorem 9. Consider the following:

(1)

$$
\begin{aligned}
\sum_{m=1}^{n-1} \sigma_{1,1}(m ; 2) \sigma_{9,1}(n-m ; 2) & \\
= & \frac{31}{5528} \sigma_{11}(n)-\frac{227}{60808} \sigma_{11,1}(n ; 2)-\frac{1}{24} \sigma_{9,1}(n ; 2) \\
& \quad-\frac{511}{264} \sigma_{1,1}(n ; 2)+\frac{1365}{691} \tau(n)+\frac{26880}{691} \tau\left(\frac{n}{2}\right),
\end{aligned}
$$

$$
\begin{aligned}
\sum_{m=1}^{n-1} \sigma_{3,1}(m ; 2) \sigma_{7,1}(n-m ; 2) & \\
= & \frac{17}{44224} \sigma_{11}(n)-\frac{79}{221120} \sigma_{11,1}(n ; 2)+\frac{7}{240} \sigma_{7,1}(n ; 2) \\
& \quad+\frac{127}{480} \sigma_{3,1}(n ; 2)-\frac{203}{691} \tau(n)-\frac{4928}{691} \tau\left(\frac{n}{2}\right),
\end{aligned}
$$


(3)

$$
\begin{aligned}
\sum_{m=1}^{n-1} \sigma_{5,1}(m ; 2) \sigma_{5,1}(n-m ; 2) \\
=\frac{1}{5528} \sigma_{11}(n)-\frac{61}{348264} \sigma_{11,1}(n ; 2) \\
\quad-\frac{31}{252} \sigma_{5,1}(n ; 2)+\frac{85}{691} \tau(n)+\frac{2560}{691} \tau\left(\frac{n}{2}\right) .
\end{aligned}
$$

Theorem 10. Consider the following:

(1)

$$
\begin{aligned}
\sum_{m=1}^{n-1} \sigma_{1,1}(m ; 2) \sigma_{11,1}(n-m ; 2) & \\
= & \frac{691}{174752} \sigma_{13}(n)-\frac{1885739}{715609440} \sigma_{13,1}(n ; 2)-\frac{1}{24} \sigma_{11,1}(n ; 2) \\
& \quad+\frac{1414477}{65520} \sigma_{1,1}(n ; 2)-\frac{891}{86} c_{14,1}(n)-\frac{8625}{762} c_{14,2}(n),
\end{aligned}
$$

$$
\begin{aligned}
\sum_{m=1}^{n-1} \sigma_{3,1}(m ; 2) \sigma_{9,1}(n-m ; 2) & \\
= & \frac{31}{174752} \sigma_{13}(n)-\frac{4769}{28834080} \sigma_{13,1}(n ; 2)+\frac{7}{240} \sigma_{9,1} \\
& \times(n ; 2)-\frac{511}{264} \sigma_{3,1}(n ; 2)-\frac{81}{86} c_{14,1}(n)+\frac{245}{254} c_{14,2}(n),
\end{aligned}
$$

$$
\begin{aligned}
\sum_{m=1}^{n-1} \sigma_{5,1}(m ; 2) \sigma_{7,1}(n-m ; 2) \\
=\frac{17}{349504} \sigma_{13}(n)-\frac{5249}{110093760} \sigma_{13,1}(n ; 2)-\frac{31}{504} \sigma_{7,1}(n ; 2) \\
\quad+\frac{127}{480} \sigma_{5,1}(n ; 2)-\frac{9}{86} c_{14,1}(n)-\frac{25}{254} c_{14,2}(n) .
\end{aligned}
$$

\section{Proof of Theorems}

In his series of eighteen papers published between 1858 and 1865, Joseph Liouville (1809-1882) stated without proof several elementary arithmetic formulae. One of these is the following formula.

Proposition 11 (see [10, page 112]). Let $f: \mathbb{Z} \rightarrow \mathbb{C}$ be an even function. Let $n \in \mathbb{N}$. Then onen obtains

$$
\sum_{\substack{(a, b, x, y) \in \mathbb{N}^{4} \\ a x+b y=n \\ a, b \text { odd }}}(f(a-b)-f(a+b))
$$

$$
\begin{aligned}
& =f(0)\left(\sigma_{1}^{*}(n)-\sigma_{0}^{*}(n)\right)-\frac{1}{2} \sum_{\begin{array}{c}
d \in \mathbb{N} \\
d \mid n \\
d \text { even }
\end{array}} d f(d) \\
& -2 \sum_{\substack{d \in \mathbb{N} \\
d \mid n \\
d \text { odd } \begin{array}{c}
t \leq t<d \\
\text { teven }
\end{array}}} f(t)
\end{aligned}
$$

Now we are ready to prove our theorems in Section 1.

Proof of Theorem 3. We apply $f(x)=x^{2 k}(k \geq 1)$ in Proposition 11. Then the left-hand side is

$$
\begin{aligned}
& \sum_{\substack{(a, b, x, y) \in \mathbb{N}^{4} \\
a x+b y=n \\
a, b \text { odd }}}(a-b)^{2 k}-(a+b)^{2 k} \\
& =\sum_{\substack{(a, b, x, y) \in \mathbb{N}^{4} \\
a x+b y=n \\
a, b \text { odd }}}\left(\sum_{r=0}^{2 k}\left(\begin{array}{c}
2 k \\
r
\end{array}\right)(-1)^{r} a^{2 k-r} b^{r}\right. \\
& \left.-\sum_{r=0}^{2 k}\left(\begin{array}{c}
2 k \\
r
\end{array}\right) a^{2 k-r} b^{r}\right) \\
& =-2 \sum_{\substack{(a, b, x, y) \in \mathbb{N}^{4} \\
a x+b y=n \\
a, b \text { odd }}} \sum_{\substack{r=0 \\
r \text { odd }}}^{2 k}\left(\begin{array}{c}
2 k \\
r
\end{array}\right) a^{2 k-r} b^{r} \\
& =-2 \sum_{s=0}^{k-1}\left(\begin{array}{c}
2 k \\
2 s+1
\end{array}\right) \sum_{\substack{(a, b, x, y) \in \mathbb{N}^{4} \\
a x+b y=n \\
a, b \text { odd }}} a^{2 k-2 s-1} b^{2 s+1} \\
& =-2 \sum_{s=0}^{k-1}\left(\begin{array}{c}
2 k \\
2 s+1
\end{array}\right) \\
& \times \sum_{m=1}^{n-1}\left(\sum_{\substack{a \mid m \\
2 \nmid a}} a^{2 k-2 s-1}\right)\left(\sum_{\substack{b \mid(n-m) \\
2 \nmid b}} b^{2 s+1}\right) \\
& =-2 \sum_{m=1}^{n-1} \sum_{s=0}^{k-1}\left(\begin{array}{c}
2 k \\
2 s+1
\end{array}\right) \\
& \times \sigma_{2 k-2 s-1,1(m ; 2) \sigma_{2 s+1,1}(n-m ; 2)} .
\end{aligned}
$$

On the other hand by using Bernoulli's identity [10, page 42],

$$
\sum_{l=1}^{n-1} l^{k}=\frac{1}{k+1} \sum_{j=0}^{k}\left(\begin{array}{c}
k+1 \\
j
\end{array}\right) B_{j} n^{k+1-j},
$$


the right-hand side is

$$
\begin{aligned}
& \sum_{\substack{(a, b, x, y) \in \mathbb{N}^{4} \\
a x+b y=n \\
a, b \text { odd }}}(a-b)^{2 k}-(a+b)^{2 k} \\
& =\sum_{\substack{(a, b, x, y) \in \mathbb{N}^{4} \\
a x+b y=n \\
a, b \text { odd }}}\left(\sum_{r=0}^{2 k}\left(\begin{array}{c}
2 k \\
r
\end{array}\right)(-1)^{r} a^{2 k-r} b^{r}\right. \\
& \left.-\sum_{r=0}^{2 k}\left(\begin{array}{c}
2 k \\
r
\end{array}\right) a^{2 k-r} b^{r}\right) \\
& =-2 \sum_{\substack{(a, b, x, y) \in \mathbb{N}^{4} \\
a x+b y=n \\
r \text { odd } \\
a, b \text { odd }}} \sum_{r=0}^{2 k}\left(\begin{array}{c}
2 k \\
r
\end{array}\right) a^{2 k-r} b^{r} \\
& =-2 \sum_{s=0}^{k-1}\left(\begin{array}{c}
2 k \\
2 s+1
\end{array}\right) \sum_{\substack{(a, b, x, y) \in \mathbb{N}^{4} \\
a x+b y=n \\
a, b \text { odd }}} a^{2 k-2 s-1} b^{2 s+1} \\
& =-2 \sum_{s=0}^{k-1}\left(\begin{array}{c}
2 k \\
2 s+1
\end{array}\right) \\
& \times \sum_{m=1}^{n-1}\left(\sum_{\substack{a \mid m \\
2 \nmid a}} a^{2 k-2 s-1}\right)\left(\sum_{\substack{b \mid(n-m) \\
2 \nmid b}} b^{2 s+1}\right) \\
& =-2 \sum_{m=1}^{n-1} \sum_{s=0}^{k-1}\left(\begin{array}{c}
2 k \\
2 s+1
\end{array}\right) \\
& \times \sigma_{2 k-2 s-1,1(m ; 2) \sigma_{2 s+1,1}(n-m ; 2)} .
\end{aligned}
$$

After dividing both sides by -2 we get

$$
2^{2 k-1} \sigma_{2 k+1}\left(\frac{n}{2}\right)+\frac{1}{4 k+2} \sum_{i=0}^{k} \widetilde{B}_{2 k-2 i}\left(\begin{array}{c}
2 k+1 \\
2 i+1
\end{array}\right) \sigma_{2 i+1,1}(n ; 2)
$$

Remark 12. When $f$ is a constant function in Proposition 11, it is a trivial formula such that

$$
\sigma_{1}(n)=2 \sigma_{1}^{*}(n)-\sigma_{1,1}(n ; 2)
$$

Corollary 13. For $k \geq 1$,

$$
\sum_{r=0}^{2 k+1} \sum_{j=0}^{2 k+1-r}\left(\begin{array}{c}
2 k+1 \\
j, r, 2 k+1-r-j
\end{array}\right) 2^{j} B_{j}=0 .
$$

Proof. Applying $n=1$ in Theorem 3, our resultis proved.
Corollary 14. Let $N \in \mathbb{N}$. Then one has

$$
\begin{aligned}
& \sum_{s=0}^{k-1}\left(\begin{array}{c}
2 k \\
2 s+1
\end{array}\right) \sum_{\substack{m=1 \\
2 \nmid m}}^{2 N} \sigma_{2 k-2 s-1}(m) \sigma_{2 s+1}(2 N-m) \\
& =2^{2 k-1} \sigma_{2 k+1}^{*}(N) .
\end{aligned}
$$

Proof. Let $n=2 \mathrm{~N}$ in Theorem 3. Then the left-hand side is

$$
\begin{aligned}
& \sum_{s=0}^{k-1}\left(\begin{array}{c}
2 k \\
2 s+1
\end{array}\right) \sum_{m=1}^{2 N-1} \sigma_{2 k-2 s-1,1}(m ; 2) \sigma_{2 s+1,1}(2 N-m ; 2) \\
& =\sum_{s=0}^{k-1}\left(\begin{array}{c}
2 k \\
2 s+1
\end{array}\right) \\
& \times\left[\sum_{\substack{m=1 \\
2 \mid m}}^{2 N-1} \sigma_{2 k-2 s-1,1}(m ; 2) \sigma_{2 s+1,1}(2 N-m ; 2)\right. \\
& \left.+\sum_{\substack{m=1 \\
2 \nmid m}}^{2 N-1} \sigma_{2 k-2 s-1,1}(m ; 2) \sigma_{2 s+1,1}(2 N-m ; 2)\right] \\
& =\sum_{s=0}^{k-1}\left(\begin{array}{c}
2 k \\
2 s+1
\end{array}\right) \\
& \times\left[\sum_{m=1}^{N-1} \sigma_{2 k-2 s-1,1}(2 m ; 2) \sigma_{2 s+1 ; 2}(2 N-2 m)\right. \\
& \left.+\sum_{\substack{m=1 \\
2 \nmid m}}^{2 N-1} \sigma_{2 k-2 s-1,1}(m ; 2) \sigma_{2 s+1,1}(2 N-m ; 2)\right] \\
& =\sum_{s=0}^{k-1}\left(\begin{array}{c}
2 k \\
2 s+1
\end{array}\right) \sum_{m=1}^{N-1} \sigma_{2 k-2 s-1,1}(m ; 2) \sigma_{2 s+1,1}(N-m ; 2) \\
& +\sum_{s=0}^{k-1}\left(\begin{array}{c}
2 k \\
2 s+1
\end{array}\right) \sum_{\substack{m=1 \\
2 \nmid m}}^{2 N} \sigma_{2 k-2 s-1}(m) \sigma_{2 s+1}(2 N-m) .
\end{aligned}
$$

The right-hand side of Theorem 3 for $n=2 N$ is

$$
\begin{aligned}
& 2^{2 k-1} \sigma_{2 k+1}(N)+\frac{1}{4 k+2} \sum_{i=0}^{k}\left(\begin{array}{c}
2 k+1 \\
2 i+1
\end{array}\right) \widetilde{B}_{2 k-2 i} \sigma_{2 i+1,1}(2 N ; 1) \\
& =2^{2 k-1} \sigma_{2 k+1}(N)+\frac{1}{4 k+2} \\
& \quad \times \sum_{i=0}^{k}\left(\begin{array}{c}
2 k+1 \\
2 i+1
\end{array}\right) \widetilde{B}_{2 k-2 i} \sigma_{2 i+1,1}(N ; 2)
\end{aligned}
$$




$$
\begin{aligned}
= & 2^{2 k-1} \sigma_{2 k+1}(N)-2^{2 k-1} \sigma_{2 k+1}\left(\frac{N}{2}\right) \\
& +\sum_{i=0}^{k-1}\left(\begin{array}{c}
2 k \\
2 i+1
\end{array}\right) \sum_{m=1}^{N-1} \sigma_{2 k-2 i-1,1}(m ; 2) \sigma_{2 i+1,1}(N-m ; 2) .
\end{aligned}
$$

Since $\sigma_{s}^{*}(N)=\sigma_{s}(N)-\sigma_{s}(N / 2)$, we are done.

Remark 15. The above result is also in [8, Theorem 3.4], but we do not use the combinatoric convolution sums of $\sigma_{k}^{*}$ functions but odd divisor function.

Corollary 16. For the odd prime p case, one has

$$
\begin{aligned}
& \sum_{s=0}^{k-1}\left(\begin{array}{c}
2 k \\
2 s+1
\end{array}\right) \sum_{m=1}^{p-1} \sigma_{2 k-2 s-1,1}(m ; 2) \sigma_{2 s+1,1}(p-m ; 2) \\
& \quad=\frac{1}{4 k+2} \sum_{r=0}^{2 k+1} \sum_{j=0}^{2 k+1-r}\left(\begin{array}{c}
2 k+1 \\
j, r, 2 k+1-j-r
\end{array}\right) 2^{j} p^{r} B_{j} .
\end{aligned}
$$

Proof. In Theorem 3,

$$
\begin{aligned}
& \sum_{s=0}^{k-1}\left(\begin{array}{c}
2 k \\
2 s+1
\end{array}\right) \sum_{m=1}^{p-1} \sigma_{2 k-2 s-1,1}(m ; 2) \sigma_{2 s+1,1}(p-m ; 2) \\
& \quad=\frac{1}{4 k+2} \sum_{s=0}^{k} \widetilde{B}_{2 k-2 s}\left(\begin{array}{c}
2 k+1 \\
2 s+1
\end{array}\right) \sigma_{2 s+1,1}(p ; 2) \\
& \quad=\frac{1}{4 k+2} \sum_{r=0}^{2 k+1} \widetilde{B}_{2 k-r+1}\left(\begin{array}{c}
2 k+1 \\
r
\end{array}\right) \sigma_{r}(p) \\
& \quad=\frac{1}{4 k+2} \sum_{r=0}^{2 k+1} \sum_{j=0}^{2 k+1-r}\left(\begin{array}{c}
2 k+1 \\
j, r, 2 k+1-j-r
\end{array}\right) 2^{j} B_{j}\left(1+p^{r}\right) \\
& =\frac{1}{4 k+2} \sum_{r=0}^{2 k+1} \sum_{j=0}^{2 k+1-r}\left(\begin{array}{c}
2 k+1 \\
j, r, 2 k+1-j-r
\end{array}\right) 2^{j} p^{r} B_{j}
\end{aligned}
$$

by Corollary 13.

Proof of Theorem 4. Note that

$$
\begin{gathered}
\widetilde{\sigma}_{s}(n)=\sigma_{s}(n)-2^{s+1} \sigma_{s}\left(\frac{n}{2}\right), \\
\sigma_{s, 1}(n ; 2)=\sigma_{s}(n)-2^{s} \sigma_{s}\left(\frac{n}{2}\right) .
\end{gathered}
$$

We reconsider Proposition 1 as the last one in the previous line:

$$
\begin{array}{r}
\sum_{s=0}^{k-1}\left(\begin{array}{c}
2 k \\
2 s+1
\end{array}\right) \sum_{m=1}^{n-1} \sigma_{2 k-2 s-1}\left(\frac{m}{2}\right) \sigma_{2 s+1}\left(\frac{n-m}{2}\right) \\
=\frac{2 k+3}{4 k+2} \sigma_{2 k+1}\left(\frac{n}{2}\right)+\left(\frac{k}{6}-\frac{n}{2}\right) \sigma_{2 k-1}\left(\frac{n}{2}\right) \\
+\frac{1}{2 k+1} \sum_{j=2}^{k}\left(\begin{array}{c}
2 k+1 \\
2 j
\end{array}\right) B_{2 j} \sigma_{2 k+1-2 j}\left(\frac{n}{2}\right) .
\end{array}
$$

Thus,

$$
\begin{aligned}
\sum_{s=0}^{k-1}\left(\begin{array}{c}
2 k \\
2 s+1
\end{array}\right) \sum_{m=1}^{n-1} \widetilde{\sigma}_{2 k-2 s-1}(m) \widetilde{\sigma}_{2 s+1}(n-m) \\
=\sum_{s=0}^{k-1}\left(\begin{array}{c}
2 k \\
2 s+1
\end{array}\right) \sum_{m=1}^{n-1}\left(\sigma_{2 k-2 s-1}(m)-2^{2 k-2 s} \sigma_{2 k-2 s-1}\left(\frac{m}{2}\right)\right) \\
\quad \times\left(\sigma_{2 s+1}(n-m)-2^{2 s+2} \sigma_{2 s+1}\left(\frac{n-m}{2}\right)\right) \\
=2 \sum_{s=0}^{k-1}\left(\begin{array}{c}
2 k \\
2 s+1
\end{array}\right) \sum_{m=1}^{n-1} \sigma_{2 k-2 s-1,1}(m ; 2) \sigma_{2 s+1,1}(n-m ; 2) \\
\quad-\sum_{s=0}^{k-1}\left(\begin{array}{c}
2 k \\
2 s+1
\end{array}\right) \sum_{m=1}^{n-1} \sigma_{2 k-2 s-1}(m) \sigma_{2 s+1}(n-m) \\
\quad+2^{2 k+1} \sum_{s=0}^{k-1}\left(\begin{array}{c}
2 k \\
2 s+1
\end{array}\right) \sum_{m=1}^{n-1} \sigma_{2 k-2 s-1}\left(\frac{m}{2}\right) \sigma_{2 s+1}\left(\frac{n-m}{2}\right) \\
=-\frac{1}{2} \widetilde{\sigma}_{2 k+1}(n)+\left(n-\frac{k}{2}\right) \widetilde{\sigma}_{2 k-1}(n)+\frac{1}{4 k+2} \\
\quad+\sum_{i=0}^{k-2}\left(\begin{array}{c}
2 k+1 \\
2 i+1
\end{array}\right)\left(\widetilde{B}_{2 k-2 i}+\left(2^{2 k-2 i}-2\right) B_{2 k-2 i}\right) \sigma_{2 s+1}(n) \\
+\frac{1}{4 k+2} \sum_{i=0}^{k-2}\left(\begin{array}{c}
2 k+1 \\
2 i+1
\end{array}\right)\left(\widetilde{B}_{2 k-2 i}-2^{2 k-2 i} B_{2 k-2 i}\right) \widetilde{\sigma}_{2 i+1}(n)
\end{aligned}
$$

by Proposition 1, Theorem 3, and (53).

The following examples show us that the coefficients of cusp forms disappear in the combinatorial convolution sum for the weights 12 and 14, explicitly.

Example 17. Consider the case of Theorem 3 for $k=5$. The left-hand side is

$$
\begin{aligned}
& \sum_{s=0}^{4}\left(\begin{array}{c}
10 \\
2 s+1
\end{array}\right) \sum_{m=1}^{n-1} \sigma_{9-2 s, 1}(m ; 2) \sigma_{2 s+1}(n-m ; 2) \\
& =20 \sum_{m=1}^{n-1} \sigma_{1,1}(m ; 2) \sigma_{1,1}(n-m ; 2) \\
& \quad+240 \sum_{m=1}^{n-1} \sigma_{3,1}(m ; 2) \sigma_{7,1}(n-m ; 2) \\
& \quad+252 \sum_{m=1}^{n-1} \sigma_{5,1}(m ; 2) \sigma_{5,1}(n-m ; 2)
\end{aligned}
$$

When we put the formula in Theorem 9 in the above, the coefficients of $\tau(n)$ and $\tau(n / 2)$ are zero. 
Example 18. The left-hand side of Theorem 3 when $k=6$ is

$$
\begin{aligned}
& 24 \sum_{m=1}^{n-1} \sigma_{1,1}(m ; 2) \sigma_{11,1}(n-m ; 2) \\
& +440 \sum_{m=1}^{n-1} \sigma_{3,1}(m ; 2) \sigma_{9,1}(n-m ; 2) \\
& +1584 \sum_{m=1}^{n-1} \sigma_{5,1}(m ; 2) \sigma_{7,1}(n-m ; 2) .
\end{aligned}
$$

By applying Theorem 10 to the above, one can check that our theorem is true and the coefficients $c_{14,1}(n)$ and $c_{14,2}(n)$ of cusp forms disappear.

\section{Conflict of Interests}

The authors declare that there is no conflict of interests regarding the publication of this paper.

\section{References}

[1] S. Ramanujan, "On certain arithmetical functions," Transactions of the Cambridge Philosophical Society, vol. 22, pp. 159-184, 1916.

[2] S. Alaca and K. S. Williams, "Evaluation of the convolution sums $\sum_{l+6 m=n} \sigma(l) \sigma(m)$ and $\sum_{2 l+3 m=n} \sigma(l) \sigma(m)$, , Journal of Number Theory, vol. 124, no. 2, pp. 491-510, 2007.

[3] H. Hahn, "Convolution sums of some functions on divisors," The Rocky Mountain Journal of Mathematics, vol. 37, no. 5, pp. 1593-1622, 2007.

[4] J. W. L. Glaisher, "Expressions for the five powers of the series in which the coefficients are the sums of the divisors of the exponents," Messenger of Mathematics, vol. 15, pp. 33-36, 1885.

[5] J. W. L. Glaisher, "On certain sums of products of quantities depending upon the divisors of a number," Messenger of Mathematics, vol. 15, pp. 1-20, 1885.

[6] J. W. L. Glaisher, "On the square of the series in which the coefficients are the sums of the divisors of the exponents," Messenger of Mathematics, vol. 14, pp. 156-163, 1884.

[7] B. Cho, D. Kim, and H. Park, "Evaluation of a certain combinatorial convolution sum in higher level cases," Journal of Mathematical Analysis and Applications, vol. 406, no. 1, pp. 203210, 2013.

[8] D. Kim and A. Bayad, "Convolution identities for twisted Eisenstein series and twisted divisor functions," Fixed Point Theory and Applications, vol. 2013, article 81, 2013.

[9] D. Kim, A. Kim, and A. Sankaranarayanan, "Bernoulli numbers, convolution sums and congruences of coefficients for certain generating functions," Journal of Inequalities and Applications, vol. 2013, article 225, 2013.

[10] K. S. Williams, Number Theory in the Spirit of Liouville, vol. 76 of London Mathematical Society Student Texts, Cambridge University Press, Cambridge, UK, 2011. 


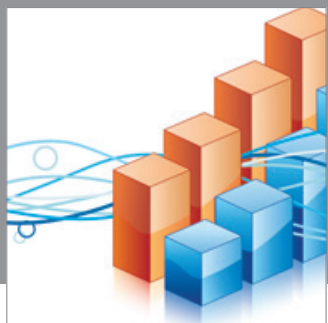

Advances in

Operations Research

mansans

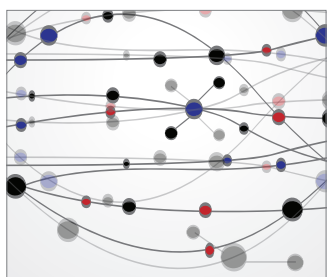

The Scientific World Journal
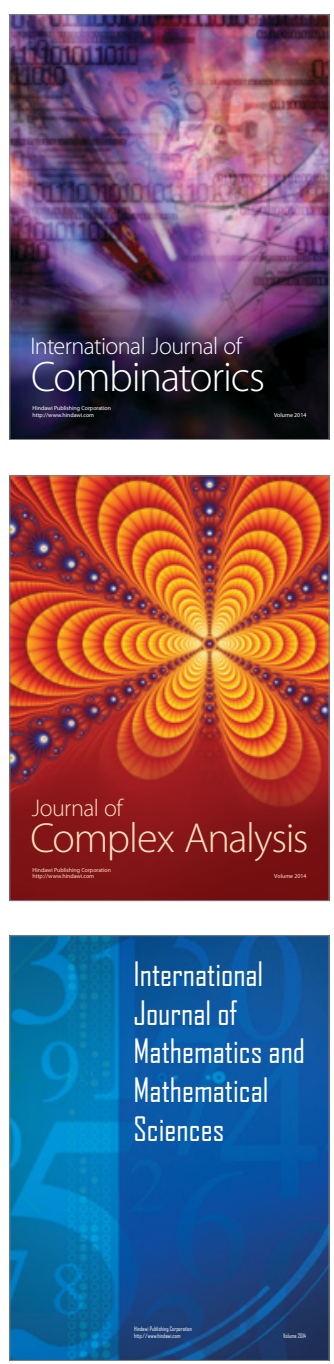
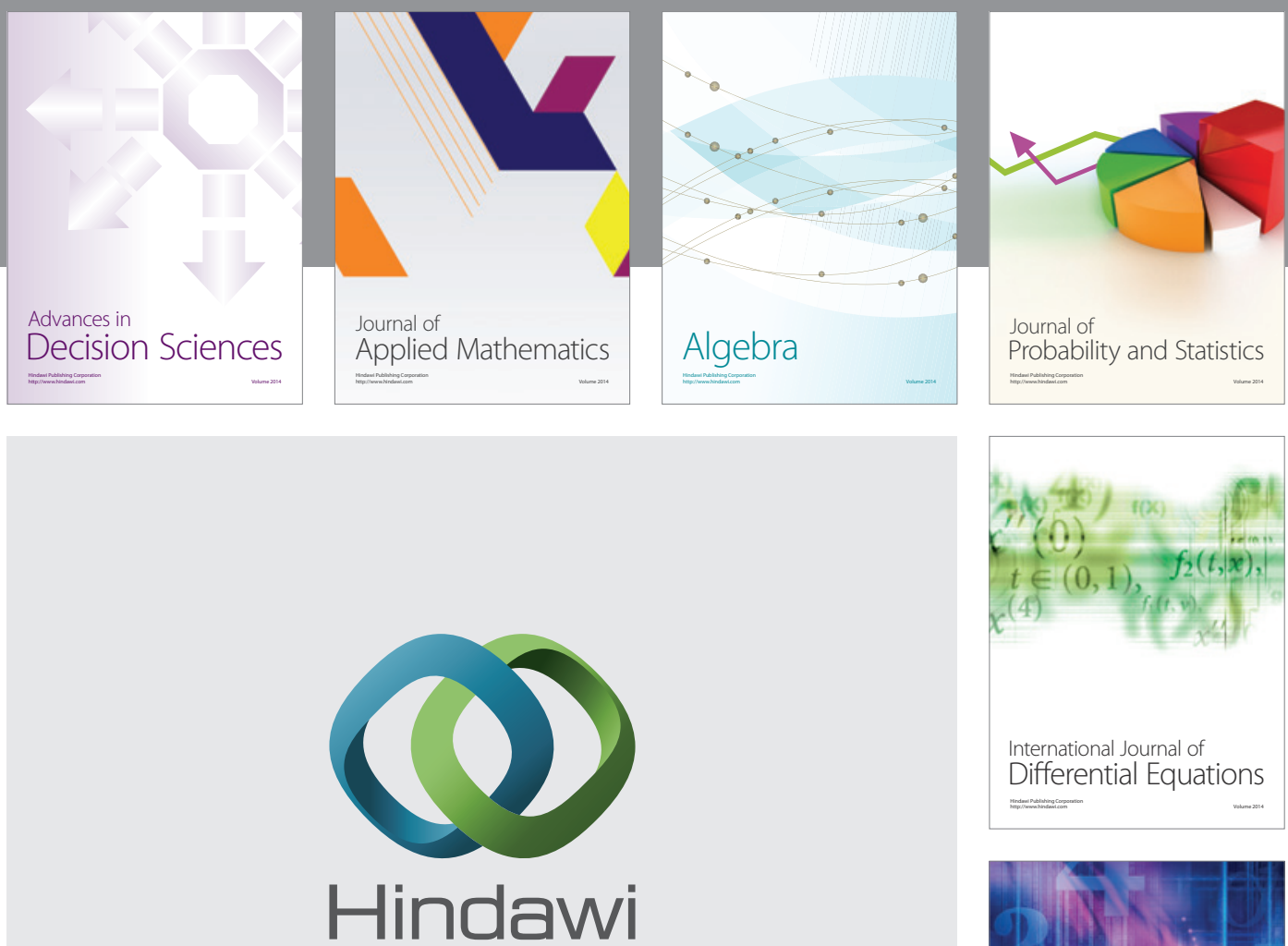

Submit your manuscripts at http://www.hindawi.com
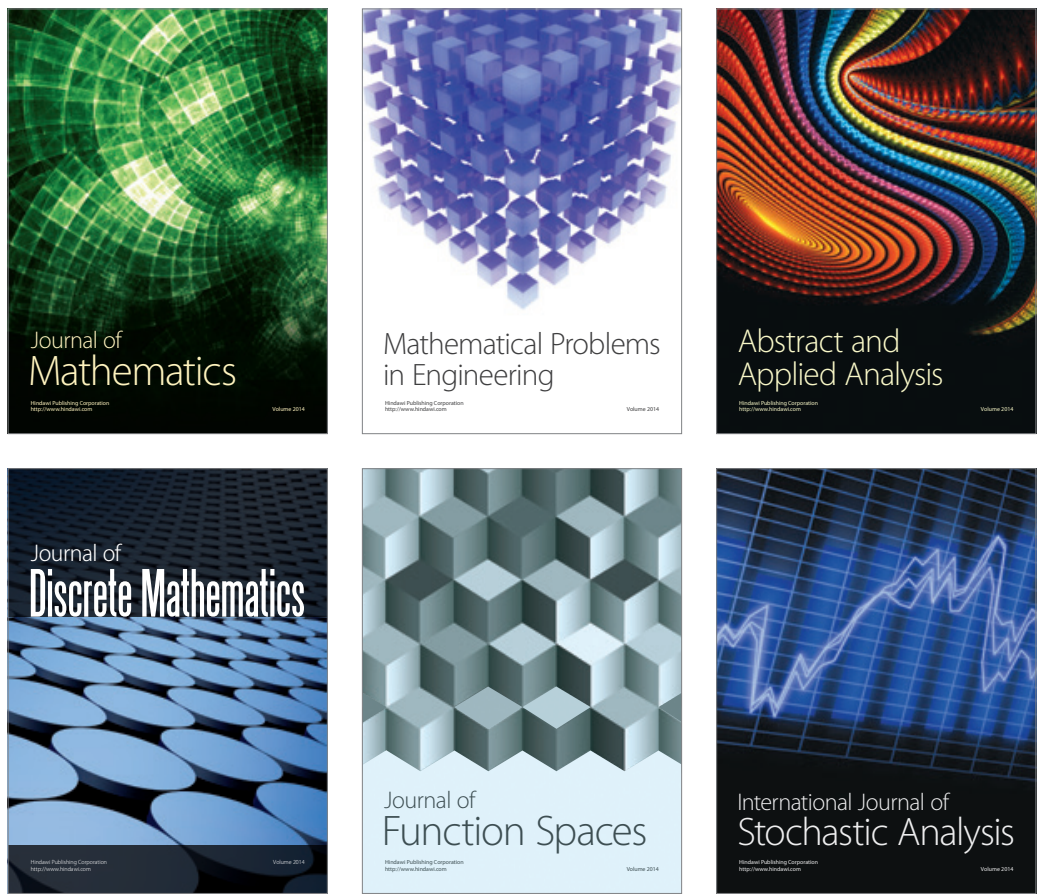

Journal of

Function Spaces

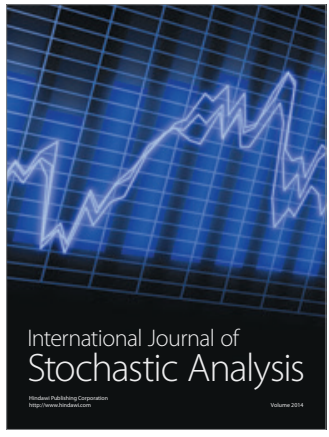

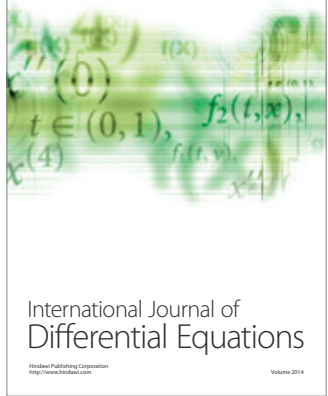
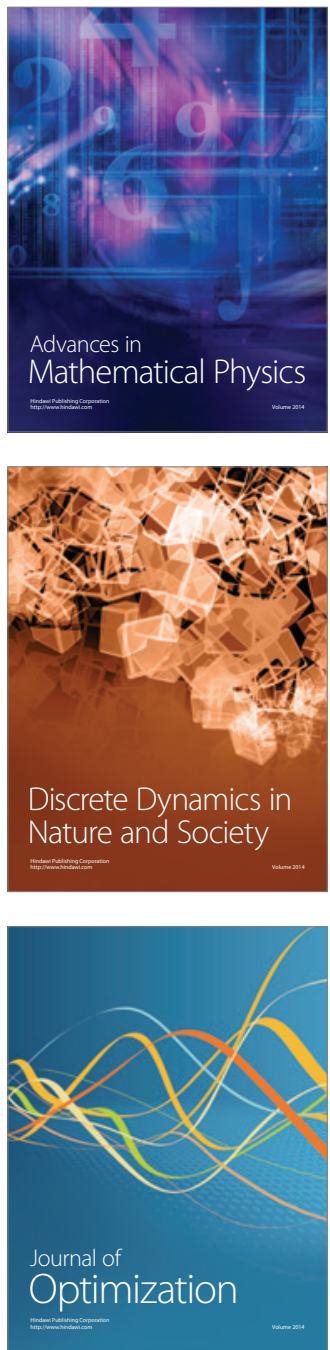